\title{
Maximum Spreading for Liquid Drop Impacting on Solid Surface
}

\author{
Gangtao Liang*, Yang Chen, Liuzhu Chen, Shengqiang Shen
}

Key Laboratory of Ocean Energy Utilization and Energy Conservation of Ministry of Education, School of Energy and Power Engineering, Dalian University of Technology, Dalian 116024, China

\footnotetext{
*Author to whom correspondence should be addressed; E-mail: gtliang@dlut.edu.cn
} 
Table S1 Summary of predicting methods for maximum spreading factor.

\begin{tabular}{|c|c|c|}
\hline Author(s) & Models or correlations & Remarks \\
\hline Jones $^{9}$ & $\beta_{\max }=\left(\frac{4}{3} R e^{1 / 4}\right)^{1 / 2}$ & $\begin{array}{l}\text { Neglecting surface } \\
\text { tension effects. }\end{array}$ \\
\hline $\begin{array}{l}\text { Kurabayashi- } \\
\text { Yang }^{14}\end{array}$ & $\frac{W e}{2}=\frac{3}{2} \beta_{\max }^{2}\left[1+\frac{3 W e}{R e}\left(\beta_{\max }^{2} \ln \beta_{\max }-\frac{\beta_{\max }^{2}-1}{2}\right)\left(\frac{\mu_{f}}{\mu_{w}}\right)^{0.14}\right]-6$ & $\begin{array}{l}\text { Involving viscosity of } \\
\text { solid surface, later } \\
\text { improved by Healy et } \\
\text { al. }^{15} \text { using a correction } \\
\text { factor, }(45 / \alpha)^{0.241} \text {. }\end{array}$ \\
\hline Madejski $^{10}$ & $\frac{3 \beta_{\max }^{2}}{W e}+\frac{1}{\operatorname{Re}}\left(\frac{\beta_{\max }}{1.2941}\right)^{5}=1$ & $\begin{array}{l}\text { Novel energy balance } \\
\text { model incorporating } \\
\text { viscous dissipation and } \\
\text { surface tension effects. }\end{array}$ \\
\hline Collings et al. ${ }^{11}$ & $\beta_{\max }=\left(\frac{W e}{6}\right)^{1 / 2}$ & $\begin{array}{l}\text { Neglecting viscous } \\
\text { dissipation. }\end{array}$ \\
\hline $\begin{array}{l}\text { Chandra and } \\
\text { Avedisian }^{16}\end{array}$ & $\frac{3 W e}{2 R e} \beta_{\max }^{4}+\left(1-\cos \alpha_{a}\right) \beta_{\max }^{2}-\left(\frac{W e}{3}+4\right)=0$ & $\begin{array}{l}\text { Involving advancing } \\
\text { contact angle. }\end{array}$ \\
\hline \multirow{2}{*}{$\begin{array}{l}\text { Bennett and } \\
\text { Poulikakos }^{12}\end{array}$} & $3\left[\left(1-\cos \alpha_{e}\right) \beta_{\max }^{2}-4\right]+1$ & \multirow{2}{*}{$\begin{array}{l}\text { Modified model based on } \\
\text { Madejski }^{10} \text {, using } \\
\text { equilibrium contact angle. }\end{array}$} \\
\hline & $W e \quad \operatorname{Re}(1.2941)$ & \\
\hline Asai et al. ${ }^{37}$ & $\beta_{\max }=1+0.48 W e^{1 / 2} \exp \left(-1.48 W e^{0.22} R e^{-0.21}\right)$ & Empirical correlation. \\
\hline $\begin{array}{l}\text { Scheller and } \\
\text { Bousfield }^{28}\end{array}$ & $\beta_{\max }=0.61\left(\operatorname{Re} W e^{1 / 2}\right)^{0.166}$ & Empirical correlation. \\
\hline $\begin{array}{l}\text { Marmanis and } \\
\text { Thoroddsen }\end{array}$ & $\beta_{\max } \propto\left(\operatorname{Re} W e^{1 / 2}\right)^{1 / 2}$ & $\begin{array}{l}\text { Theoretical model using } \\
\text { natural free oscillation } \\
\text { period. }\end{array}$ \\
\hline $\begin{array}{l}\text { Pasandideh-Fard } \\
\text { et al. }\end{array}$ & $\beta_{\max }=\left[\frac{W e+12}{3\left(1-\cos \alpha_{a}\right)+4\left(W e R e^{-1 / 2}\right)}\right]^{1 / 2}$ & $\begin{array}{l}\text { Energy balance model } \\
\text { incorporating advancing } \\
\text { contact angle, } \alpha_{a} .\end{array}$ \\
\hline Mao et al. ${ }^{13}$ & {$\left[\frac{1}{4}\left(1-\cos \alpha_{e}\right)+0.2 \frac{W e^{0.83}}{R e^{0.33}}\right] \beta_{\max }^{3}-\left(\frac{W e}{12}+1\right) \beta_{\max }+\frac{2}{3}=0$} & Semiempirical model \\
\hline
\end{tabular}


Clanet et al. ${ }^{30} \quad \beta_{\max } \propto W e^{1 / 4}$

Clanet et al. ${ }^{30} \quad \beta_{\max } \propto R e^{1 / 5} f\left(W e R e^{-4 / 5}\right)$

Ukiwe and

Kwok $^{18}$

Fedorchenko et $a .^{31}$

Bayer and

Megaridis $^{25}$

Vadillo et al. ${ }^{19}$

$(W e+12)$

$\beta_{\max } \propto R e^{1 / 5}$

$\beta_{\text {max }}=0.72\left(\operatorname{Re} W e^{1 / 2}\right)^{0.14}$

Roisman $^{7}$

Eggers et $a l^{32}$

Laan et al. ${ }^{33} \quad \beta_{\max }=\frac{\operatorname{Re}^{1 / 5}\left(W e R e^{-2 / 5}\right)^{1 / 2}}{1.24+\left(W e R e^{-2 / 5}\right)^{1 / 2}}$

Seo et al. ${ }^{29}$ $\beta_{\text {max }} \propto R e^{1 / 5} f\left(W e R e^{-2 / 5}\right)$

$$
\beta_{\max }=0.87 R e^{1 / 5}-0.40 R e^{2 / 5} W e^{-1 / 2}
$$

$$
\beta_{\max }=1.27\left(\operatorname{Re} W e^{1 / 2}\right)^{0.122}
$$

Momentum balance model using impact capillary length, for low viscosity and low wettability conditions.

Capillary regime is based on effective gravity theory.

Modified model based on Pasandideh-Fard et al. ${ }^{17}$, using Young contact angle, $\alpha_{Y}$, approximating to advancing contact angle, $\alpha_{a}$, for smooth surface.

Within viscous regime.

Empirical correlation.

Substituting the Young contact angle, $\alpha_{Y}$, in Ukiwe and Kwok $^{18}$ with dynamic contact angle, $\alpha_{d}$.

Semiempirical correlation involving a viscous boundary layer.

Capillary regime is determined by balance between inertia and surface tension.

Smoothed transition between viscous regime and capillary regime.

Empirical correlation. 
$\begin{array}{lll}\text { Wildeman et al. } & 38 & \beta_{\max }=\sqrt{\frac{4}{1-\cos \alpha_{a}}\left(\frac{1}{24} W e+1\right)} \quad \text { For } W e>30 .\end{array}$

Table S2 Liquid properties and experimental conditions.

\begin{tabular}{ccccccc}
\hline Fluid & $\sigma[\mathrm{N} / \mathrm{m}]$ & $\mu[\mathrm{Pa} \cdot \mathrm{s}]$ & $\rho\left[\mathrm{kg} / \mathrm{m}^{3}\right]$ & $\alpha_{e}\left[{ }^{\circ}\right]$ & $d_{\text {drop }}[\mathrm{mm}]$ & $v_{\text {drop }}[\mathrm{m} / \mathrm{s}]$ \\
\hline Water & 0.072 & 0.001003 & 1000 & 87 & 2.62 & $0.39-1.41$ \\
Butanol & 0.0201 & 0.00295 & 810 & 26 & 2.03 & $0.39-1.48$ \\
Ethanol & 0.0224 & 0.0012 & 790 & 23 & 1.98 & $0.39-1.41$ \\
\hline
\end{tabular}

Table S3 Summary of select predicting methods for assessment.

\begin{tabular}{ll}
\hline Author(s) & Models or correlations \\
\hline Scheller and Bousfield $^{28}$ & $\beta_{\text {max }}=0.61\left(\operatorname{Re} W e^{1 / 2}\right)^{0.166}$ \\
Bayer and Megaridis $^{25}$ & $\beta_{\text {max }}=0.72\left({\left.\operatorname{Re} W e^{1 / 2}\right)^{0.14}}\right.$ \\
Roisman $^{7}$ & $\beta_{\text {max }}=0.87 \operatorname{Re}^{1 / 5}-0.40 \operatorname{Re}^{2 / 5} W e^{-1 / 2}$ \\
Laan et al. $^{33}$ & $\beta_{\text {max }}=\frac{\operatorname{Re}^{1 / 5}\left(W e e^{-2 / 5}\right)^{1 / 2}}{1.24+\left(W e e^{-2 / 5}\right)^{1 / 2}}$ \\
\hline
\end{tabular}

Table S4 Measured data of maximum spreading factor included in the consolidated database.

Author(s) Test fluid(s) Surface material Contact angle $\left[{ }^{\circ}\right] \quad$ Data points

$\begin{array}{llll}\text { Pasandideh-Fard } & \text { Water } & \text { Steel } & \alpha_{a}=110^{\circ}\end{array}$

Ford and Furmidge $^{39}$

Vadillo et al. ${ }^{19}$
Water

Mixtures of Crystal silicon water-glycerol and glass
Beeswax,

cellulose acetate, $\quad \alpha_{a}=27-111^{\circ} \quad 15$

wafer with silicon $\alpha_{e}=5-90^{\circ}, \alpha_{a}=$ oxide layer

$15-107^{\circ}$ 6 


\begin{tabular}{|c|c|c|c|c|}
\hline Cheng $^{40}$ & Water & Bituminous coal & L & 17 \\
\hline An and $\mathrm{Lee}^{41}$ & $\begin{array}{l}\text { Water and } \\
\text { mixtures of } \\
\text { water-glycerol }\end{array}$ & $\begin{array}{l}\text { Glass, stainless } \\
\text { steel and } \\
\text { parafilm-M }\end{array}$ & $\alpha_{e}=37.9-108.4^{\circ}$ & 24 \\
\hline Lee $e t a .^{21}$ & $\begin{array}{l}\text { Ethanol, water } \\
\text { and glycerol }\end{array}$ & $\begin{array}{l}\text { Aluminum, steel } \\
\text { and parafilm }\end{array}$ & $\begin{array}{l}\alpha_{e}=0-109.6^{\circ}, \alpha_{a}= \\
0-115^{\circ}\end{array}$ & 93 \\
\hline $\begin{array}{l}\text { Zhang and } \\
\text { Basaran }^{42}\end{array}$ & $\begin{array}{l}\text { Water and } \\
\text { aqueous } \\
\text { surfactant } \\
\text { solutions }\end{array}$ & Glass & $\alpha_{e}=54^{\circ}$ & 11 \\
\hline Kim et al. ${ }^{43}$ & Water & Polycarbonate & $\ldots$ & 3 \\
\hline Kim and Chun $^{44}$ & Water & $\begin{array}{l}\text { Polycarbonate } \\
\text { and silicon oxide }\end{array}$ & $\alpha_{e}=58.6-87.4^{\circ}$ & 5 \\
\hline Mao et al. ${ }^{13}$ & Water & $\begin{array}{l}\text { Paraffin wax, } \\
\text { stainless steel and } \\
\text { glass }\end{array}$ & $\alpha_{e}=37-97^{\circ}$ & 21 \\
\hline Present & $\begin{array}{l}\text { Water, butanol } \\
\text { and ethanol }\end{array}$ & Stainless steel & $\alpha_{e}=23-87^{\circ}$ & 15 \\
\hline Total & & & & 213 \\
\hline
\end{tabular}

Table S5 Comparison of individual $\beta_{\max }$ databases with predictions of select previous methods and new proposed formulas.

\begin{tabular}{lllllll}
\hline $\begin{array}{l}\text { Database } \\
\text { authors }\end{array}$ & $\begin{array}{l}\text { Scheller and } \\
\text { Bousfield }^{28}\end{array}$ & $\begin{array}{l}\text { Bayer and } \\
\text { Megaridis }^{25}\end{array}$ & Roisman $^{7}$ & ${\text { Laan } \text { et al. }^{33}}^{\text {Eq. (3) }}$ & Eq. (4) \\
\hline $\begin{array}{l}\text { Pasandideh-Fard } \\
\text { et al. }^{17}\end{array}$ & 24.9 & 15.7 & 9.6 & 6.4 & 15.6 & 7.9 \\
$\begin{array}{l}\text { Ford and } \\
\text { Furmidge }\end{array}$ & 7.3 & 13.1 & 7.4 & 9.1 & 9.1 & 8.3 \\
$\begin{array}{l}\text { Vadillo } \text { et al. }^{19} \\
\text { Cheng }^{40}\end{array}$ & 20.0 & 15.3 & 33.0 & 18.7 & 18.0 & 20.8
\end{tabular}




\begin{tabular}{|c|c|c|c|c|c|c|}
\hline An and Lee ${ }^{41}$ & 7.0 & 8.7 & 3.6 & 7.0 & 7.6 & 119.3 \\
\hline Lee $e t a .^{21}$ & 18.5 & 18.1 & 17.4 & 11.6 & 15.7 & 9.8 \\
\hline $\begin{array}{l}\text { Zhang and } \\
\text { Basaran }^{42}\end{array}$ & 15.3 & 14.0 & 14.8 & 4.3 & 9.0 & 1.7 \\
\hline Kim et $a l .{ }^{43}$ & 3.4 & 13.8 & 4.4 & 2.5 & 7.9 & 20.1 \\
\hline Kim and Chun ${ }^{44}$ & 16.0 & 12.5 & 10.5 & 4.5 & 11.4 & 4.4 \\
\hline Mao et al. ${ }^{13}$ & 21.2 & 18.8 & 8.2 & 4.2 & 15.9 & 5.7 \\
\hline Present & 24.8 & 24.1 & 19.3 & 16.9 & 24.6 & 8.9 \\
\hline Total & 15.9 & 15.8 & 13.3 & 9.5 & 13.6 & 21.6 \\
\hline
\end{tabular}

